\title{
Analytic Probabilistic Safety Analysis Under Severe Uncertainty
}

\author{
Jonathan Sadeghi ${ }^{1}$, Marco De Angelis ${ }^{2}$, and Edoardo Patelli ${ }^{3}$ \\ ${ }^{1} \mathrm{PhD}$ candidate, Institute for Risk and Uncertainty, Chadwick Building, University of Liverpool, \\ Peach Street, Liverpool L69 7ZF, United Kingdom \\ ${ }^{2}$ Post-doctoral researcher, Institute for Risk and Uncertainty, Chadwick Building, University of \\ Liverpool, Peach Street, Liverpool L69 7ZF, United Kingdom \\ ${ }^{3}$ Senior Lecturer, Institute for Risk and Uncertainty, Chadwick Building, University of Liverpool, \\ Peach Street, Liverpool L69 7ZF, United Kingdom, edoardo.patelli@liverpool.ac.uk
}

\begin{abstract}
Exact analytic expressions are given to evaluate the reliability of systems consisting of components, connected in parallel or series, subject to imprecise failure distributions. We also proposed a simplified version of the first order reliability method to deal with imprecision. This development allows engineers to evaluate the reliability of systems without having to resort to optimisation techniques and/or Monte Carlo simulation. In addition, this framework does not need to assume a distribution for the epistemic uncertainty, which permits a robust analysis even with limited data. In this way, the approach removes a significant barrier to the modelling of epistemic uncertainties in industrial probabilistic safety analysis workflows.
\end{abstract}

\section{INTRODUCTION}

Probabilistic safety analysis (PSA) was first introduced in the 1970s as a means of establishing the probability of a certain amount of radiation release to the environment from a nuclear structure. It is perceived to address many of the weaknesses of deterministic analysis (Modarres and Kim 2010). For example, deterministic analysis relies heavily on engineering conservatism which could 
be difficult to quantify in practice. In addition, it is not always clear what the most conservative value for a particular parameter is when performing a black box analysis.

In recent years, techniques from the area of imprecise probability have been increasingly applied to Probabilistic Safety Analysis studies in academic literature (Karanki et al. 2009) (Beer and Patelli 2015). Imprecise probabilities offer a natural framework to model uncertainty due to lack of knowledge (epistemic uncertainty). Epistemic uncertainty is particularly important in the nuclear industry where there is often a lack of sufficient data to completely model relevant phenomena. This uncertainty can be modelled as interval uncertainty in the parameters of traditional probability distributions, which is known as probability bounds analysis, where the imprecise distributions themselves are referred to as probability boxes (Ferson et al. 2003). This approach strikes a pragmatic compromise between engineering conservatism and overly optimistic analyses. However, the techniques proposed usually require sophisticated simulation techniques (Patelli 2016; Patelli et al. 2017a). For example, to propagate uncertainty through a complex black box model computationally expensive Monte Carlo or optimisation methods are used (Patelli et al. 2015). Although efficient simulation approaches for dealing with imprecision have recently been proposed (e.g. using Line Sampling (de Angelis et al. 2015), Subset simulation (Patelli et al. 2011) and tools to deal with probability boxes (Patelli et al. 2014; Patelli et al. 2017b; Faes et al. 2019)), their use in the nuclear industry is not yet widespread. In Ref. (Le Duy et al. 2010) recommendations are made for how available data can be used to define probability boxes. In Ref. (Qiu et al. 2008) approximate results were derived for structural systems where the First Order Reliability Method (FORM) could be applied. In the United States the nuclear regulator (Budnitz et al. 1985) refers to the work of Kennedy who provides many analytic relationships to establish the fragility curve for a containment with a conventional probabilistic treatment (Kennedy et al. 1980). The effect of epistemic uncertainty in PSA with conventional probability was considered in (Prinja et al. 2017; Sun and Yao 2008).

In (conventional) structural probabilistic safety analysis often the relations used are simple analytic expressions which, in contrast to the methods based on imprecise probability, allow the 
failure probability of the system to be computed with no Monte Carlo simulation at all. This offers two significant advantages. Firstly, the computational time required to complete the calculations is greatly reduced, which allows projects to be completed on shorter timescales and less money to be spent on High Performance Computing. Secondly, the time of engineers is saved as they are not required to spend large amounts of time programming Monte Carlo simulations, which reduces expenditure for their employer, and consequently benefits the industry as a whole.

In this paper, we will propose imprecise probabilistic analogues to many of the probabilistic formulae proposed in Kennedy's paper which have become standard expressions used in probabilistic safety analysis. In this way, we hope to unite the conventional literature which is applied to PSA in industry with relatively recent developments in imprecise probability. The analysis will make extensive use of the probability boxes introduced in probability bounds theory. We will demonstrate how to establish the fragility curve of a system when components are connected in parallel or series, and when the failures of the components may have unknown dependencies. We will demonstrate how to establish a probability box fragility curve when the product of random variables must be considered. Then, we will also demonstrate how this can be used to calculate the failure probability when there is additional imprecision in the load distribution. We will also consider the implications of the imprecise First Order Reliability Method (FORM), and show how we can analytically obtain results from a simplified calculation when the exact reliability index is difficult to obtain. All of the above are particularly useful when combined with an event tree to e.g. yield the expected radiation release to the environment or to calculate the reliability of complex plant. The proposed approach considers only independence of components or complete lack of information on dependence. In fact, the framework of imprecise probability enables the consideration of correlations between events, for example via convex sets or copula functions. This would be a useful future generalisation of the work in this paper, as any additional information available will allow the bounds on the probability of failure to be tightened.

The merit of this approach is that the entire fragility curve can be constructed by one analyst using conventional spreadsheet packages, without the requirement to use complicated simulation 
techniques which would require large amounts of time spent programming by the analyst. Therefore the benefits of traditional PSA approaches are retained whilst also obtaining the advantages of using probability bounds theory.

In Section 2 an brief overview is presented of a typical PSA calculation used to determine the fragility curve of a system. In Section 3 we propose analogues to the expressions from Section 2 using probability bounds analysis. In Section 4 a simple example is presented. In Section 4 we show how similar methods can be applied to obtain partial information about a more complex system. In Section 5 a brief summary is given.

\section{PROBABILISTIC SAFETY ANALYSIS}

Probabilistic safety analysis is broken down into three levels. Level 1 PSA studies the reactor and determines accident sequences which are likely to result in a release from the reactor pressure vessel. Level 2 considers the containment structure, and how likely this is to fail in an accident. This is done by creating a fragility curve for the containment, which quantifies the failure probability at a particular load. Level 3 PSA combines the information produced by level 1 and level 2 PSA to provide the probability of radiation release to the environment (Commission et al. 2005).

In PSA level 2 the main goal is to establish the fragility curve of a (nuclear) structure (Pellissetti et al. 2017). In seismic hazard analysis the fragility curve expresses the failure probability of the structure as a function of the peak ground acceleration. This can then be used to conduct safety analysis once the conditions inside the reactor (the 'source term') and the external conditions are known (Sundararajan 2012).

The fragility of a system is its probability of failure conditioned on a particular load. Therefore, in the context of this section of the paper, bounds on failure probabilities may be taken as bounds on fragilities. For a system, $S$, of components, $c_{i}$, connected in series (i.e. the system will fail if one component fails) the fragility of the system, $f(s \mid a)$, at a damage measure $a$ (i.e. the peak ground acceleration) is given by

$$
f(s \mid a)=1-\prod_{c_{i} \subset S}\left[1-f\left(c_{i} \mid a\right)\right]
$$


when the fragilities of the individual components are independently distributed (Kennedy et al. 1980).

If the dependence is not known then the value of $f(s \mid a)$ given by Eqn. (1) is an upper bound which, for the small probabilities relevant to this type of analysis, approaches the value given by the more general Boole's inequality, in what is known as the rare event approximation (Collet 1996). Boole's inequality can be used to calculate an upper bound on the probability that at least one event from a set of events occurs, i.e. the probability that a series system fails, when the dependence between different events is unknown. The Fréchet inequalities are similar upper and lower bounds that apply to the union and intersection of events when no information is available about the dependence of events (Rüschendorf 1991).

Boole's inequality is equal to the right hand side of the Fréchet inequality for the upper bound of the union of $n$ events:

$$
\max \left(P\left(A_{1}\right), \ldots, P\left(A_{n}\right)\right) \leq P\left(\bigcup_{i=1}^{n} A_{i}\right) \leq \min \left(1, P\left(A_{1}\right)+\ldots+P\left(A_{n}\right)\right)
$$

The other Fréchet inequality (which applies for components connected in parallel) being

$$
\max \left(0, P\left(A_{1}\right)+\ldots+P\left(A_{n}\right)-(n-1)\right) \leq P\left(\bigcap_{i=1}^{n} A_{i}\right) \leq \min \left(P\left(A_{1}\right), \ldots, P\left(A_{n}\right)\right)
$$

Note that both Boole's inequality and the Fréchet inequalities are conservative bounds which should be used when the dependence between failure events is unknown.

If the fragilities of the components are independently distributed and the components are connected in parallel (i.e. the system has redundancy and fails if every component fails) then the system's fragility is given by

$$
f(s \mid a)=\prod_{c_{i} \subset S}\left[f\left(c_{i} \mid a\right)\right] .
$$

If the dependence between component fragilities is not known then the value of $f(s \mid a)$ given by Eqn. (4) is an upper bound (Kennedy et al. 1980). These formulae can also be applied to 
connected systems which form super systems, in which case the unknown dependence versions on the equations should be used (Kennedy et al. 1980).

In probabilistic safety analysis $f\left(c_{i} \mid a\right)$ is usually modelled as a log normally distributed random variable, because the physical quantities being modelled must be greater than zero, i.e.

$$
f\left(c_{i} \mid a\right)=\phi\left(\frac{\log \left(\frac{a}{\beta_{i}}\right)}{\sigma_{i}}\right)
$$

where $\beta_{i}$ represents the median failure value and $\sigma_{i}$ is the logarithmic standard deviation of component $c_{i}$, and $\phi$ is the cumulative distribution function (CDF) of a standard normal variable. Typically in probabilistic safety analysis aleatory uncertainty can be distinguished from epistemic uncertainty by modelling the $\beta$ for any particular component as a lognormally distributed random variable with parameters $\beta_{e}$ and $\sigma_{e}$. Hence the outer distribution (i.e. Eqn. (5), with logarithmic standard deviation $\sigma_{a}$ ) will describe aleatory uncertainty, and epistemic uncertainty is modelled by the nested distribution (i.e. the inner distribution, the CDF over $\beta$, with parameters $\beta_{e}$ and $\sigma_{e}$ ).

In order to allow this model to be used for computation, typically the mean distribution is obtained (more widely known as the 'composite' distribution), which is also log-normally distributed. This is an averaged distribution obtained by combining the aleatory uncertainty (i.e. $\sigma_{a}$ from the outer distribution) and the epistemic uncertainty (our uncertainty in the distribution parameters, $\left.\sigma_{e}\right)$ (Kim et al. 2010). For the composite distribution, the logarithmic standard deviation, $\sigma_{c}$, is the euclidean norm of the two lognormal logarithmic standard deviations, i.e. $\sigma_{c}=\sqrt{\sigma_{a}^{2}+\sigma_{e}^{2}}$ and the median is simply the median of the inner (epistemic) distribution, $\beta_{c}=\beta_{e}$ (a detailed derivation is provided in Ref. (Kaplan et al. 1994)). This distribution is assumed to be conservative, since it approaches the asymptotic values in the tails of the distributions described by the extrema of the epistemic distribution (Kennedy et al. 1980). However, in many cases there may be insufficient data to truly know that our epistemic uncertainty is log-normally distributed.

Figure 1 shows an example of a composite distribution compared to the median fragility curve 
and the 5th and 95th percentiles of the epistemic uncertainty. As discussed, the mean curve approaches the extreme outer distributions' tails (obtained by taking $\beta$ from the 5th and 95th percentiles of the nested epistemic distribution and $\sigma=\sigma_{a}$ ). Clearly the median curve could not be used for this purpose as it does not adequately describe the range of our belief in the peak ground acceleration.

\section{PROBABILITY BOUNDS ANALYSIS}

\section{Fragility Curve}

Let us consider the fragility distribution for a general component given by Eqn. (1). Instead of considering $\beta_{i}$ as a random variable and finding the composite distribution we will instead consider uncertainty in $\beta_{i}$ and $\sigma_{i}$ as intervals. This enables the random variables to be converted into probability boxes, since probability boxes are nothing more than cumulative distribution functions with interval imprecision on the distribution parameters. This framework is attractive for several reasons. Firstly, we do not need to assume a distribution for our epistemic uncertainty, which permits a robust analysis even with limited data. Secondly, instead of having to find the composite distribution we can simply find the envelope of our distributions. Note that uniform distributions are conceptually different from interval incertitude, since a uniform distribution specifies that each value in the support of the distribution is equally likely, whereas an interval describes lack of knowledge in a set-like manner, without implications for the likelihood of different elements within the set. Furthermore, note that using probability distributions to represent epistemic uncertainty has been shown to have undesirable consequences (Balch 2016) (Balch et al. 2017).

In Appendix I we show that if $\beta_{i} \in\left[\underline{\beta_{i}}, \overline{\beta_{i}}\right]$ and $\sigma_{i} \in\left[\underline{\sigma_{i}}, \overline{\sigma_{i}}\right]$ then the distributional probability box can be converted to a distribution free probability box where the upper bound of the fragility is given by

$$
\overline{f\left(c_{i} \mid a\right)}=\phi\left(\frac{\log \left(\frac{a}{\beta_{i}}\right)-\left|\log \left(\frac{a}{\beta_{i}}\right)\right|}{2 \overline{\sigma_{i}}}+\frac{\log \left(\frac{a}{\beta_{i}}\right)+\left|\log \left(\frac{a}{\beta_{i}}\right)\right|}{2 \underline{\sigma_{i}}}\right),
$$


and the lower bound of the fragility is given by

$$
\underline{f\left(c_{i} \mid a\right)}=\phi\left(\frac{\log \left(\frac{a}{\overline{\beta_{i}}}\right)+\left|\log \left(\frac{a}{\overline{\beta_{i}}}\right)\right|}{2 \overline{\sigma_{i}}}+\frac{\log \left(\frac{a}{\overline{\beta_{i}}}\right)-\left|\log \left(\frac{a}{\overline{\beta_{i}}}\right)\right|}{2 \underline{\sigma_{i}}}\right),
$$

where the $|\cdot|$ operator represents the absolute value of a quantity. These bounds are shown in Figure 2. In general converting distributional probability boxes to distribution free probability boxes results in loss of information (Alvarez et al. 2017). However, in this case Eqn. (6) and Eqn. (7) are a result of taking the natural extension of Eqn. (5) and therefore the values obtained will be the tightest bounds possible, so in the specific case of Eqn. (6) and Eqn. (7) there is no consequence to making the conversion. The other results in this section provide the tightest possible bound in the case of unknown dependence, since we simply apply a Fréchet inequality. Note that the other results in the paper, after this section, do not make use of the conversion used in this section, in order to avoid the potential information loss.

For systems containing components in series or parallel, when the component failures are known to be independent, the fragility can be calculated by using Eqn. (1) and Eqn. (4), respectively. Alternatively, if the failure dependence is unknown we can use the relevant Fréchet inequality, Eqns. (2) and (3), to yield the fragility. Alternatively, in the case of unknown failure dependence, the rare event approximation (described in Section 2) can be used to justify the application of Eqn. (1) and Eqn. (4) which will be accurate in the tails of the distributions (i.e. for rare events).

Therefore, using the natural interval extension of Eqn. (2) with Eqn. (6) and Eqn. (7) it can be shown that, for components in series, the probability of failure at a particular ground motion, $a$, with certainty falls in the interval given by

$$
f(s \mid a) \in\left[\max _{i}\left[\underline{f\left(c_{i} \mid a\right)}\right], \min \left(1, \sum_{i=1}^{n}\left[\overline{f\left(c_{i} \mid a\right)}\right]\right)\right],
$$


i.e.

$$
\begin{array}{r}
f(s \mid a) \in\left[\max _{i}\left[\phi\left(\frac{\log \left(\frac{a}{\overline{\beta_{i}}}\right)+\left|\log \left(\frac{a}{\overline{\beta_{i}}}\right)\right|}{2 \overline{\sigma_{i}}}+\frac{\log \left(\frac{a}{\overline{\beta_{i}}}\right)-\left|\log \left(\frac{a}{\overline{\beta_{i}}}\right)\right|}{2 \underline{\sigma_{i}}}\right)\right],\right. \\
\left.\min \left(1, \sum_{i=1}^{n}\left[\phi\left(\frac{\log \left(\frac{a}{\beta_{i}}\right)-\left|\log \left(\frac{a}{\beta_{i}}\right)\right|}{2 \overline{\sigma_{i}}}+\frac{\log \left(\frac{a}{\beta_{i}}\right)+\left|\log \left(\frac{a}{\beta_{i}}\right)\right|}{2 \underline{\sigma_{i}}}\right)\right]\right)\right]
\end{array}
$$

\section{Product of log-normally distributed random variables}

Often the fragility curve for a component must be established by considering the product of a number of random variables with lognormal distributions. If this is the case then the probability bounds analysis approach can be extended to allow us to find the relevant fragility curve. To demonstrate, consider a general random variable $d$ which is given by the product of other random variables, i.e.

$$
d=q \frac{a^{r} b^{s}}{c^{t}}
$$

where $a, b$ and $c$ are lognormal random variables and $q, r, s$ and $t$ are constants. It is clear that $d$ will be lognormally distributed with median $\beta_{d}=q \frac{\beta_{a}^{r} \beta_{b}^{s}}{\beta_{c}^{t}}$, and logarithmic standard deviation $\sigma_{d}^{2}=r^{2} \sigma_{a}^{2}+s^{2} \sigma_{b}^{2}+t^{2} \sigma_{c}^{2}$ (Kennedy et al. 1980).

In the case of interval imprecision in the distribution parameters of $a, b$ and $c$ we can obtain

$$
\overline{\beta_{d}}=q \cdot \frac{\overline{\beta_{a}^{r}} \cdot \overline{\beta_{b}^{s}}}{\underline{\beta}_{c}^{t}}
$$

and

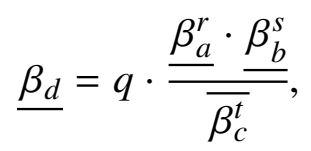

by using the endpoint formulae for interval multiplication (Moore et al. 2009) with knowledge of the support of the distribution parameters. The logarithmic standard deviation can be obtained 
from

$$
\overline{\sigma_{d}^{2}}=r^{2} \overline{\sigma_{a}^{2}}+s^{2} \overline{\sigma_{b}^{2}}+t^{2} \overline{\sigma_{c}^{2}}
$$

and

$$
\underline{\sigma_{d}^{2}}=r^{2} \underline{\sigma_{a}^{2}}+s^{2} \underline{\sigma_{b}^{2}}+t^{2} \underline{\sigma_{c}^{2}}
$$

by taking the interval extension of the expression stated above for the case of no interval imprecision.

This is principally of use when computing the response factor, $F$, which can be expressed as the product of a number of response factors applying to different pieces of equipment and processes (for example damping effects or modelling effects), i.e. $F=\prod_{i} F_{i}$. The $F_{i}$ are modelled as lognormal random variables and may have interval imprecision in the median (Sundararajan 2012).

\section{Failure Probability}

Consider a system which fails when the load exceeds the strength. For a general load distribution the failure probability is given by

$$
P_{f}=-\int_{0}^{\infty} \frac{d H(a)}{d a} f(s \mid a) d a
$$

where $H(a)$ is the seismic hazard curve (i.e. the probability that the 'load' exceeds a certain value in a particular unit of time, which usually takes the form of the complement of a CDF since it must be monotonically decreasing, and the probability cannot exceed 1) (American Society of Civil Engineers 2005). When $H(a)$ and $f(s \mid a)$ are both log normally distributed, it is simple to solve Eqn. (15) by transforming the integral (Kapur and Lamberson 1977). However, in general this integral is not solvable analytically and it cannot be solved analytically when the fragility curve is replaced with the distribution free probability boxes derived in the previous section.

Therefore to derive bounds on the failure probability of systems subject to distributional probability box loads and fragilities we will apply Fréchet bounds and interval arithmetic to well known results obtained by solving Eqn. (15) for common probability distributions.

For example consider the case where the probability distribution function of the load, $\frac{d H(a)}{d a}$, 
is log-normally distributed with parameters $\beta_{l}$ and $\sigma_{l}$ and the fragility, $f(s \mid a)$, is lognormally distributed with parameters $\beta_{i}$ and $\sigma_{i}$. In this case, the failure probability can be evaluated as

$$
P_{f}=\phi\left(-\frac{\log \beta_{i}-\log \beta_{l}}{\sqrt{\sigma_{i}^{2}+\sigma_{l}^{2}}}\right) .
$$

A plot of distributions used in Eqn. (16) with example parameters is shown in Figure 3.

To calculate an upper bound on the failure probability for a series system we evaluate the maximum and minimum of Eqn. (16) with $\beta_{l} \in\left[\underline{\beta_{l}}, \bar{\beta}_{l}\right], \sigma_{l} \in\left[\underline{\sigma_{l}}, \bar{\sigma}_{l}\right], \beta_{i} \in\left[\underline{\beta_{i}}, \bar{\beta}_{i}\right], \sigma_{i} \in\left[\underline{\sigma_{i}}, \bar{\sigma}_{i}\right]$ and Eqn. (2). Analogously, for components in parallel a similar result can be obtained from Eqn. (3). For simple systems these bounds provide useful analytic quantification of the reliability of the system under epistemic uncertainty. However, for more complex systems the bounds are usually not analytically calculable and hence numerical integration may be necessary (e.g. (Samaniego 2007), (Patelli et al. 2017b), (Feng et al. 2016)).

It is likely that there is uncertainty in $\beta_{l}$ and $\sigma_{l}$. If this is the case then the analysis can be made robust using an uncertainty quantification approach for the load distribution which is analogous to the approach used for the fragility.

In some works, such as ASCE 43-05 (Braverman et al. 2007), the hazard curve has been modelled as a power law, since this is a good approximation to the Cauchy-Pareto complementary cumulative distribution function (Kennedy 2011). Such an equation takes the form of

$$
H(a)=k_{1} a^{-K_{H}}
$$

where $k_{1}$ and $K_{H}$ are positive fitted constants. $K_{H}$ represents the slope of the mean seismic hazard curve when plotted on log-log scale. With a log-normal fragility in the parametrisation used in this paper, the failure probability for a single component is given by

$$
P_{f}=H\left(\beta_{i}\right) \exp \frac{\left(K_{H} \sigma_{i}\right)^{2}}{2}
$$


- Series system with unknown dependence; Lognormal load and Strength:

$$
\overline{P_{f}}=\sum_{c_{i} \subset S} \max \left[\phi\left(-\frac{\log \underline{\beta_{i}}-\log \bar{\beta}_{l}}{\sqrt{\bar{\sigma}_{i}^{2}+\bar{\sigma}_{l}^{2}}}\right), \phi\left(-\frac{\log \underline{\beta_{i}}-\log \bar{\beta}_{l}}{\sqrt{{\underline{\sigma_{i}^{2}}}^{2}+\underline{\sigma}_{l}^{2}}}\right)\right]
$$

and

$$
\underline{P_{f}}=\max _{c_{i} \subset S}\left[\min \left[\phi\left(-\frac{\log \bar{\beta}_{i}-\log \underline{\underline{\beta_{l}}}}{\sqrt{\bar{\sigma}_{i}^{2}+\bar{\sigma}_{l}^{2}}}\right), \phi\left(-\frac{\log \bar{\beta}_{i}-\log \underline{\beta_{l}}}{\sqrt{\underline{\sigma_{i}^{2}}+\underline{\sigma}_{l}^{2}}}\right)\right]\right]
$$


- Series system with independent components (upper bound also valid for dependant rare events); Log-normal load and strength:

$$
\overline{P_{f}}=1-\prod_{c_{i} \subset S}\left[1-\max \left[\phi\left(-\frac{\log \underline{\beta_{i}}-\log \bar{\beta}_{l}}{\sqrt{\bar{\sigma}_{i}^{2}+\bar{\sigma}_{l}^{2}}}\right), \phi\left(-\frac{\log \underline{\beta_{i}}-\log \bar{\beta}_{l}}{\sqrt{{\underline{\sigma_{i}}}^{2}+\underline{\sigma}_{l}^{2}}}\right)\right]\right]
$$

and

$$
\underline{P_{f}}=1-\prod_{c_{i} \subset S}\left[1-\min \left[\phi\left(-\frac{\log \bar{\beta}_{i}-\log \underline{\beta_{l}}}{\sqrt{\bar{\sigma}_{i}^{2}+\bar{\sigma}_{l}^{2}}}\right), \phi\left(-\frac{\log \bar{\beta}_{i}-\log \underline{\beta_{l}}}{\sqrt{{\underline{\sigma_{i}}}^{2}+\underline{\sigma}_{l}^{2}}}\right)\right]\right]
$$

- Parallel system (independent components - upper bound also valid for dependant rare events); Log-normal load and strength

$$
\underline{P}_{f}=\prod_{c_{i} \subset S} \min \left[\phi\left(-\frac{\log \bar{\beta}_{i}-\log \bar{\beta}_{l}}{\sqrt{\bar{\sigma}_{i}^{2}+\bar{\sigma}_{l}^{2}}}\right), \phi\left(-\frac{\log \bar{\beta}_{i}-\log \bar{\beta}_{l}}{\sqrt{\underline{\sigma}_{i}^{2}+\bar{\sigma}_{l}^{2}}}\right)\right]
$$

and

$$
\bar{P}_{f}=\prod_{c_{i} \subset S} \max \left[\phi\left(-\frac{\log \underline{\beta}_{i}-\log \underline{\beta}}{\sqrt{\bar{\sigma}_{i}^{2}+\underline{\sigma}_{l}^{2}}}\right), \phi\left(-\frac{\log \underline{\beta_{i}}-\log \underline{\beta}_{l}}{\sqrt{\underline{\sigma}_{i}^{2}+\underline{\sigma}_{l}^{2}}}\right)\right]
$$

- Single Component; Power Law Load, with $k_{1} \in\left[\underline{k}_{1}, \bar{k}_{1}\right]$ and $K_{H} \in\left[\underline{K}_{H}, \bar{K}_{H}\right]$; Lognormal, with median $\beta \in[\underline{\beta}, \bar{\beta}]$ and logarithmic standard deviation $\sigma \in[\underline{\sigma}, \bar{\sigma}]$ :

$$
\bar{P}_{f}=\bar{k}_{1} \max \left[\underline{\beta}^{-\bar{K}_{H}} \exp \frac{\left(\bar{K}_{H} \bar{\sigma}\right)^{2}}{2}, \underline{\beta}^{-\underline{K}_{H}} \exp \frac{\left(\underline{K}_{H} \bar{\sigma}\right)^{2}}{2}\right]
$$


and conservative lower bound

$$
\underline{P}_{f}=\underline{k}_{1} \bar{\beta}^{-\bar{K}_{H}} \exp \frac{\left(\underline{K}_{H} \underline{\sigma}\right)^{2}}{2}
$$

If $\underline{K_{H}}>\frac{\log \bar{\beta}}{\underline{\sigma}^{2}}$ or $\bar{K}_{H}<\frac{\log \underline{\beta}}{\bar{\sigma}^{2}}$ a tighter lower bound is obtained from:

$$
\underline{P}_{f}=\underline{k}_{1} \min \left[\bar{\beta}^{-\underline{K}_{H}} \exp \frac{\left(\underline{K}_{H} \underline{\sigma}\right)^{2}}{2}, \bar{\beta}^{-\bar{K}_{H}} \exp \frac{\left(\bar{K}_{H} \underline{\sigma}\right)^{2}}{2}\right]
$$

- Parallel system with unknown dependence; Power Law Load, with $k_{1} \in\left[\underline{k}_{1}, \bar{k}_{1}\right]$ and $K_{H} \in\left[\underline{K}_{H}, \bar{K}_{H}\right]$ Lognormal, with median $\beta_{i} \in\left[\underline{\beta}_{i}, \bar{\beta}_{i}\right]$ and logarithmic standard deviation $\sigma_{i} \in\left[\underline{\sigma}_{i}, \bar{\sigma}_{i}\right]:$

$$
\bar{P}_{f}=\bar{k}_{1} \min _{c_{i} \subset S}\left[\max \left[\underline{\beta}_{i}^{-\bar{K}_{H}} \exp \frac{\left(\bar{K}_{H} \bar{\sigma}_{i}\right)^{2}}{2}, \underline{\beta}_{i}^{-\underline{K}_{H}} \exp \frac{\left(\underline{K}_{H} \bar{\sigma}_{i}\right)^{2}}{2}\right]\right]
$$

and

$$
\underline{P}_{f}=\underline{k}_{1} \sum_{c_{i} \subset S}\left[\bar{\beta}_{i}{ }^{-\bar{K}_{H}} \exp \frac{\left(\underline{K}_{H}{\underline{\sigma_{i}}}^{2}\right.}{2}\right]-(n-1)
$$

- Series system with unknown dependence; Power Law Load, with $k_{1} \in\left[\underline{k}_{1}, \bar{k}_{1}\right]$ and $K_{H} \in$ $\left[\underline{K}_{H}, \bar{K}_{H}\right]$ Log-normal, with median $\beta_{i} \in\left[\underline{\beta}_{i}, \bar{\beta}_{i}\right]$ and logarithmic standard deviation $\sigma_{i} \in$ $\left[\underline{\sigma}_{i}, \bar{\sigma}_{i}\right]:$

$$
\bar{P}_{f}=\bar{k}_{1} \sum_{c_{i} \subset S} \max \left[\underline{\beta}_{i}^{-\bar{K}_{H}} \exp \frac{\left(\bar{K}_{H} \bar{\sigma}_{i}\right)^{2}}{2}, \underline{\beta}_{i}^{-\underline{K}_{H}} \exp \frac{\left(\underline{K}_{H} \bar{\sigma}_{i}\right)^{2}}{2}\right]
$$


and

$$
\underline{P}_{f}=\underline{k}_{1} \max _{c_{i} \subset S}\left[\bar{\beta}_{i}^{-\bar{K}_{H}} \exp \frac{\left(\underline{K}_{H} \underline{\sigma}_{i}\right)^{2}}{2}\right]
$$

- Parallel system with independent components ( upper bound also valid for dependant rare events); Power Law Load, with $k_{1} \in\left[\underline{k}_{1}, \bar{k}_{1}\right]$ and $K_{H} \in\left[\underline{K}_{H}, \bar{K}_{H}\right]$; Log-normal, with median $\beta_{i} \in\left[\underline{\beta}_{i}, \bar{\beta}_{i}\right]$ and logarithmic standard deviation $\sigma_{i} \in\left[\underline{\sigma}_{i}, \bar{\sigma}_{i}\right]$ :

$$
\bar{P}_{f}=\prod_{c_{i} \subset S} \underline{k}_{1} \max \left[\underline{\beta}_{i}^{-\bar{K}_{H}} \exp \frac{\left(\bar{K}_{H} \bar{\sigma}_{i}\right)^{2}}{2}, \underline{\beta}_{i}^{-\underline{K}_{H}} \exp \frac{\left(\underline{K}_{H} \bar{\sigma}_{i}\right)^{2}}{2}\right]
$$

and

$$
\underline{P}_{f}=\prod_{c_{i} \subset S} \bar{k}_{1} \bar{\beta}_{i}{ }^{-\bar{K}_{H}} \exp \frac{\left(\underline{K}_{H} \underline{\sigma}_{i}\right)^{2}}{2}
$$

- Series system with independent components (upper bound also valid for dependant rare events); Power Law Load, with $k_{1} \in\left[\underline{k}_{1}, \bar{k}_{1}\right]$ and $K_{H} \in\left[\underline{K}_{H}, \bar{K}_{H}\right]$; Log-normal, with median $\beta_{i} \in\left[\underline{\beta}_{i}, \bar{\beta}_{i}\right]$ and logarithmic standard deviation $\sigma_{i} \in\left[\underline{\sigma}_{i}, \bar{\sigma}_{i}\right]$ :

$$
\bar{P}_{f}=1-\prod_{c_{i} \subset S}\left[1-\bar{k}_{1} \max \left[\underline{\beta}_{i}^{-\bar{K}_{H}} \exp \frac{\left(\bar{K}_{H} \bar{\sigma}_{i}\right)^{2}}{2}, \underline{\beta}_{i}^{-\underline{K}_{H}} \exp \frac{\left(\underline{K}_{H} \bar{\sigma}_{i}\right)^{2}}{2}\right]\right]
$$

and

$$
\underline{P}_{f}=1-\prod_{c_{i} \subset S}\left[1-\underline{k}_{1} \bar{\beta}_{i}{ }^{-\bar{K}_{H}} \exp \frac{\left(\underline{K}_{H}{\underline{\sigma_{i}}}^{2}\right.}{2}\right]
$$

\section{Imprecise FORM}

The failure probability of a structural system calculated by Eqn. (15) can be approximated by $P_{f}=\phi(-\beta)$, using the well known FORM approximation, where $\beta$ is the reliability index. The 
reliability index can be obtained from

$$
\beta=\frac{\mu_{S}-\mu_{L}}{\sigma_{L}^{2}+\sigma_{S}^{2}}
$$

where $\mu_{S}$ and $\mu_{L}$ are the mean values of the strength and load and $\sigma_{L}$ and $\sigma_{S}$ are the standard deviations of the strength and load. Note that the FORM approximation holds exactly for linear limit state functions with normally distributed strength and load, but is only an approximation when the mean and standard deviations of other distributions are used.

In (Qiu et al. 2008) this is extended to the case of probability box random variables, so that $\bar{P}_{f}=\phi(-\underline{\beta})$ and $\underline{P}_{f}=\phi(-\bar{\beta})$ where

$$
\bar{\beta}=\frac{\bar{\mu}_{S}-\underline{\mu_{L}}}{\underline{\sigma_{L}^{2}}+\underline{\sigma_{S}^{2}}},
$$

and

$$
\underline{\beta}=\frac{\underline{\mu}_{S}-\bar{\mu}_{L}}{\bar{\sigma}_{L}^{2}+\bar{\sigma}_{S}^{2}} .
$$

In more complex cases one may need to use optimisation to find the reliability index using methods derived from the Hasofer-Lind method (Jiang et al. 2017). For example, one could imagine a system which fails if the sum of many different products of probability box distributed variables falls below a threshold. However, even in these cases, we can attempt to analyse in which conditions the system is likely to fail using a simple analytical method.

Consider a load term which is the product of a constant and a random variable, i.e. $L=C L_{d}$, where $C$ is a constant and $L_{d}$ is a random variable representing the design load. The system will have a $P_{f}=0.5$ when $\beta=0$, which implies the strength to load ratio, $\gamma=\frac{\mu_{S}}{\mu_{L}}$, will be equal to 1 . Clearly, this is only the case when $C=\gamma_{d}=\frac{\mu_{S}}{\mu_{L_{d}}}$, i.e. the applied load is scaled by the strength to design load ratio (Prinja et al. 2017).

This can be trivially extended in the case of probability box variables to find an interval load 
for which $P_{f}=0.5$, i.e. $L_{0.5} \in\left[\underline{L_{0.5}}, \bar{L}_{0.5}\right]=\left[\underline{\gamma}_{d} L_{d}, \bar{\gamma}_{d} L_{d}\right.$, $]$ where

$$
\bar{\gamma}_{d}=\frac{\bar{\mu}_{S}}{\underline{\mu}_{L_{d}}},
$$

and

$$
\underline{\gamma}_{d}=\frac{\underline{\mu}_{S}}{\bar{\mu}_{L_{d}}} .
$$

Note that the standard deviation of the random variables is not involved in the calculation of this load.

\section{ILLUSTRATIVE EXAMPLES}

\section{Reliability analysis of a simple concrete containment}

To demonstrate the results described in the previous sections we will consider a modified version of an example given in (Modarres et al. 2016) with interval imprecision in the coefficient of variation of the random variables. The random variables will be modelled with lognormal distributions since lognormal distributions are commonly used to model physical quantities which must always be positive in the probabilistic safety analysis literature (Sundararajan 2012), (Kennedy et al. 1980). However note our approach could be applied to similar problems with different distribution types, and many other distributions exist to ensure positivity of random variables. The problem description will be briefly replicated in this section for clarity.

A concrete containment is a structure designed to prevent radioactive release from nuclear power plants to the environment. It is therefore important that the reliability of this structure can be determined accurately, as failing to do so could have severe consequences for the environment and the general public. During the process of determining the reliability of a containment, engineers wish to determine the relationship between applied pressure and failure probability of the containment. A simplified performance function is used to perform reliability analysis without having to run simulations on a complex finite element model. This approach is advantageous as the computational time required is significantly reduced. The approach assumes that the system will fail if the load is larger than the strength. 
The containment's strength is considered to be divided between 7 failure mode contributors, all of which may cause system failure. Therefore, this example can be treated as a system composed of 7 components (which are modelled as random variables), connected in series.

The probability of failure for the containment is given by

$$
P_{f}=\int_{S_{t}<L_{t}} f(\boldsymbol{x}) d \boldsymbol{x}
$$

where $f(\boldsymbol{x})$ is the joint probability distribution function of the random variables, $\boldsymbol{x}=\left(x_{1}, x_{2} \ldots\right)$ and $S_{t}$ and $L_{t}$ represent the strength and load terms respectively. The input parameter values assumed in this analysis were taken approximately from the original example (Modarres et al. 2016), but modified to fit lognormal variables and include some imprecision as shown in Table 1. The pressure load inside the containment, for the specific accident being considered, was taken to be lognormally distributed with mean $0.575 \mathrm{MPa}$ and standard deviation of $0.117 \mathrm{MPa}$ (such that the parameters for the fitted lognormal distribution were $\log \beta=-0.5737 \mathrm{MPa}$ and $\sigma=0.2014 \mathrm{MPa}$ ).

The fragility of the series system was bounded using Eqn. (9) and compared to the empirical CDFs obtained by randomly sampling the epistemic uncertainty. The results are shown in Figure 5.

The failure probability was calculated using Eqn. (20), since the dependence between failure modes was unknown. This resulted in a failure probability between 0.0086 and 0.0123 , which contains the precise probability of failure $\left(P_{f}=0.0122\right)$ given in (Modarres et al. 2016). This result was verified by use of double loop Monte Carlo simulation, which was performed using the same samples used to generate Figure 5 (100 epistemic samples and 1000 aleatory samples). The analytic code took 0.027 seconds to run, whilst the double loop Monte Carlo simulation took 0.16 seconds to run on an 2.9 GHz Intel Core i5 processor in MAтLAв. In addition the result from double loop Monte Carlo simulation would require more samples, and hence even greater time, to increase accuracy in the tails of the p-box to an arbitrary amount already achieved by the analytic approach.

These results reveal a good agreement with the expensive simulation procedures in a fraction (one fifth) of the time. Note that although in this case the double loop Monte Carlo was quick to 
run, this may not be true in general (such as in high dimensional cases). In addition, the Monte Carlo simulation could be one nested component in a much larger computation. Even when this is not the case, it is unrealistic to expect practising engineers to resort to double loop Monte Carlo simulation for what should be a simple design calculation, even with the inclusion of epistemic uncertainty. In practical cases it would also be necessary to consider uncertainty in the Logarithmic Mean of the random variables which can be easily accounted for given the developments in Section 3.

\section{Containment with Additive Component Strengths}

In many real systems the components' strengths may be added together, rather than combined in parallel or series. Such an example is given in (Prinja et al. 2017). This poses a challenge for analytical methods, as in general normal distributions and log normal distributions cannot be summed easily (except in limited cases such as independently distributed normal random variables). Therefore, in order to consider such systems in the imprecise PSA framework, we resort to using the imprecise FORM approximations given in (Qiu et al. 2008).

A pre-stressed concrete containment is a concrete structure designed to prevent the release of radiation from the core of a nuclear reactor to the environment. The structural reliability analysis of pre-stressed concrete containments is a key component of level 2 PSA.

In (Prinja et al. 2017) probabilistic safety analysis of a concrete containment was presented as part of a round robin international test exercise. Two experimental test cases (Sandia National Laboratories and Bhabha Atomic Research Centre) are described and the probability of failure for each containment is calculated. The experiments are compared to a cylindrical concrete containment model, where the area and strength of the concrete, rebar, tendons and liner are modelled as normally distributed random variables. In this study, we will focus on the SNL containment, but add some epistemic uncertainty to the random variables. This epistemic uncertainty could represent lack of knowledge about the materials used for yield values, or lack of knowledge about the design being considered for geometric properties. The modified properties of the Sandia National Laboratories containment are summarised in Table 2. 
The performance function of the containment is obtained as a load-strength relationship, i.e.

$$
g=\left(A_{s} F_{s}+A_{t} F_{t}+A_{l} F_{l}+A_{c} F_{c}\right)-P R .
$$

We set the applied pressure to be equal to the design pressure, scaled by a constant.

Using the strength to design load ratio method from Eqn. (31) and Eqn. (32) with

$$
\frac{\bar{\mu}_{S}}{\underline{\mu}_{L}}=\frac{\bar{\mu}_{A_{s}} \bar{\mu}_{F_{s}}+\bar{\mu}_{A_{t}} \bar{\mu}_{F_{t}}+\bar{\mu}_{A_{c}} \bar{\mu}_{F_{c}}+\bar{\mu}_{A_{l}} \bar{\mu}_{F_{l}}}{\underline{\mu}_{P_{d}} \underline{\mu_{R}}}
$$

and

$$
\frac{\underline{\mu}}{\bar{\mu}_{L}}=\frac{\underline{\mu}_{A_{s}}{ }_{F_{s}}+\underline{\mu}_{A_{t}} \underline{\mu}_{F_{t}}+\underline{\mu}_{A_{c}} \underline{\mu}_{F_{c}}+\underline{\mu}_{A_{l}} \underline{\mu}_{F_{l}}}{\bar{\mu}_{P_{d}} \bar{\mu}_{R}}
$$

we find that $P_{f}=0.5$ when $P \in\left[5.2 P_{d}, 5.24 P_{d}\right]$. In order words, because of our epistemic uncertainty in the structural properties of the system we are unsure which pressure causes $P_{f}=0.5$. Clearly the epistemic uncertainty we have considered does not significantly change the pressure at which $P_{f}=0.5$.

For a more complete understanding of the system (i.e. understanding which pressures cause large and small failure probabilities) advanced simulation methods would be necessary. This is because the strength to design-load ratio method only considers the mean values of the random variable in order to find the pressure at which the structure has $P_{f}=0.5$, and does not consider the variability of the structural components. For example, one could resort to the method proposed in Ref. (de Angelis et al. 2015), where line sampling is applied to structures with epistemic uncertainties.

\section{CONCLUSIONS}

In this paper, we have demonstrated methods to analytically propagate probability boxes in commonly used probabilistic safety analysis equations. These equations include series and parallel systems with unknown dependencies, lognormal fragility distributions and equations where lognormally distributed factors are multiplied. In addition, Power Law Load load distributions are 
considered. Crucially, we use intervals to model epistemic uncertainty in the parameters of these distributions. This enables the robust quantification of epistemic uncertainty when performing probabilistic safety analysis, particularly in an industrial context. These distributions are sufficient for the analysis of many industrial problems, but in general the imprecise probability methods proposed could be generalised to other distributions as well.

These expressions are imprecise probabilistic analogues to many of the probabilistic formulae proposed in Kennedy's paper (Kennedy et al. 1980), which have become standard expressions used in probabilistic safety analysis. We also demonstrate how similar techniques can be applied to simplified calculations involving more complex models.

Our proposed expressions enable engineers to complete essential design calculations whilst considering epistemic uncertainty, and avoid the impracticalities of double loop Monte Carlo simulation which we believe is a significant barrier to the modelling of epistemic uncertainty in many industrial probabilistic safety assessment workflows. 


\section{APPENDIX I. PROOFS}

\section{Proof of Eqns. (6) and (7)}

Firstly, note that $\phi$ is a monotonic function of its arguments, so finding the maxima and minima of Eqn. (5) is reduced to finding the maxima and minima of $\frac{\log \frac{a}{\beta_{i}}}{\sigma_{i}}$ when $\beta_{i} \in\left[\underline{\beta_{i}}, \bar{\beta}_{i}\right]$ and $\sigma_{i} \in\left[\underline{\sigma_{i}}, \bar{\sigma}_{i}\right]$. Then note that $\log \frac{a}{\bar{\beta}_{i}}<\log \frac{a}{\beta_{i}}<\log \frac{a}{\beta_{i}}$. The upper bound is found by noting that if $0<\log \frac{a}{\underline{\beta_{i}}}$ then $\frac{\log \frac{a}{\beta_{i}}}{\sigma_{i}}<\frac{\log \frac{a}{\beta_{i}}}{\underline{\sigma_{i}}}$ and if $0>\log \frac{a}{\underline{\beta_{i}}}$ then $\frac{\log \frac{a}{\beta_{i}}}{\sigma_{i}}<\frac{\log \frac{a}{\beta_{i}}}{\bar{\sigma}_{i}}$. The lower bound is found by noting that if $0<\log \frac{a}{\bar{\beta}_{i}}$ then $\frac{\log \frac{a}{\beta_{i}}}{\sigma_{i}}>\frac{\log \frac{a}{\bar{\beta}_{i}}}{\bar{\sigma}_{i}}$ and if $0>\log \frac{a}{\bar{\beta}_{i}}$ then $\frac{\log \frac{a}{\bar{\beta}_{i}}}{\sigma_{i}}>\frac{\log \frac{a}{\bar{\beta}_{i}}}{\underline{\sigma_{i}}}$.

Finally, note that it is trivial to construct a function which takes a different value above and below zero, e.g. $\frac{f_{1}(x)-\left|f_{1}(x)\right|}{c_{1}}+\frac{f_{1}(x)+\left|f_{1}(x)\right|}{c_{2}}$ is equal to $\frac{2 f_{1}(x)}{c_{2}}$ above zero and $\frac{2 f_{1}(x)}{c_{1}}$ below zero. This concludes the proof.

\section{Proof of Equations in Section 3}

The failure probability bounds for a parallel system with unknown dependencies and lognormally distributed load and strength, Eqn. (19), can be derived by applying the natural interval extension of the Fréchet inequality for the intersection, Eqn. (3), to the natural interval extension of the failure probability for a lognormal component, Eqn. (16).

Eqn. (20), the series system with unknown dependencies and lognormally distributed load and strength is derived in the same way, except this time the union Fréchet inequality (Eqn. (2)) is applied.

Eqn. (21) and Eqn. (22) can be derived in the same way by applying Eqn. (1) and Eqn. (4), respectively.

The derivation of Eqn. (23) (single component with power law load and log normal fragility) is more complex, due to repeated variables $\left(K_{H}\right)$ (Moore et al. 2009). Firstly, note that $P_{f}=$ $H\left(\beta_{i}\right) \exp \frac{\left(K_{H} \sigma_{i}\right)^{2}}{2}=k_{1} \exp \left(-K_{H} \log \beta_{i}+\frac{1}{2} K_{H}^{2} \sigma_{i}^{2}\right)$. Recall that $k_{1}>0, K_{H}>0, \beta>0$ and $\sigma>0$. Note that $P_{f}$ is monotonic in $k_{1}, \sigma_{i}$ and $\beta_{i}$, so our task is simply to find $\max _{K_{H}} \bar{k}_{1} \exp \left(-K_{H} \log \underline{\beta_{i}}+\frac{1}{2} K_{H}^{2} \bar{\sigma}_{i}^{2}\right)$ and $\min _{K_{H}} \underline{k_{l}} \exp \left(-K_{H} \log \bar{\beta}_{i}+\frac{1}{2} K_{H}^{2} \underline{\sigma}_{i}^{2}\right)$.

The function $k_{1} \exp \left(-K_{H} \log \beta_{i}+\frac{1}{2} K_{H}^{2} \sigma_{i}^{2}\right)$ is quadratic in $K_{H}$ and has a global minima in $K_{H}$ at $K_{H}=\frac{\log \beta}{\sigma^{2}}$. Clearly $\max _{K_{H}} \bar{k}_{1} \exp \left(-K_{H} \log \underline{\beta_{i}}+\frac{1}{2} K_{H}^{2} \bar{\sigma}_{i}^{2}\right)$ takes its maximum value at $\bar{K}_{H}$ or $\underline{K_{H}}$. 
Elementary interval analysis reveals that $\underline{k_{l}} \exp \left(-K_{H} \log \bar{\beta}_{i}+\frac{1}{2} K_{H}^{2} \underline{\sigma}_{i}^{2}\right)>\underline{k_{l}} \exp \left(-\bar{K}_{H} \log \bar{\beta}_{i}+\frac{1}{2} \underline{K}_{H}^{2} \underline{\sigma}_{i}^{2}\right)$. However in reality $\overline{K_{H}}$ and $K_{H}$ cannot appear in the same expression, as they represent specific values of the same quantity. A tighter bound is obtained by checking if $\underline{K_{H}}<\frac{\log \bar{\beta}_{i}}{\underline{\sigma_{i}{ }^{2}}}<\bar{K}_{H}$. If this inequality holds then the minimum occurs at $K_{H}=\frac{\log \bar{\beta}_{i}}{{\underline{\sigma_{i}}}^{2}}$. Otherwise we must check which of $\bar{K}_{H}$ and $\underline{K_{H}}$ minimises the failure probability. Then the remaining results can be obtained by applying the union or intersection Fréchet inequalities, or rare event approximation as appropriate. 


\section{ACKNOWLEDGEMENTS}

This research has been generously supported by the EPSRC Centre for Doctoral Training in Nuclear Fission - Next Generation Nuclear (Grant reference: EP/L015390/1) which is gratefully acknowledged by the authors. The authors would like to express their gratitude for insightful discussion regarding the industrial application of probabilistic safety analysis with Professor Nawal K. Prinja (Technology Director of Clean Energy, Wood plc). 


\section{REFERENCES}

Alvarez, D. A., Hurtado, J. E., and Ramírez, J. (2017). “Tighter bounds on the probability of failure than those provided by random set theory." Computers \& Structures, 189, 101-113.

American Society of Civil Engineers (2005). "Seismic design criteria for structures, systems, and components in nuclear facilities (ASCE/SEI 43-05).

Balch, M. S. (2016). “A corrector for probability dilution in satellite conjunction analysis.” 18th AIAA Non-Deterministic Approaches Conference, 1445.

Balch, M. S., Martin, R., and Ferson, S. (2017). "Satellite conjunction analysis and the false confidence theorem." arXiv preprint arXiv:1706.08565.

Beer, M. and Patelli, E. (2015). "Editorial: Engineering analysis with vague and imprecise information." Structural Safety Special Issue: Engineering Analyses with Vague and Imprecise Information, 52, Part B(0), 143 Engineering Analyses with Vague and Imprecise Information.

Braverman, J., Xu, J., Ellingwood, B., Costantino, C., Morante, R., and Hofmayer, C. (2007). "Evaluation of the seismic design criteria in ASCE/SEI standard 43-05 for application to nuclear power plants." Report no., US Nuclear Regulatory Commission, Job Code N.

Budnitz, R. J., Amico, P. J., Cornell, C. A., Hall, W. J., Kennedy, R. P., Reed, J. W., and Shinozuka, M. (1985). "Approach to the quantification of seismic margins in nuclear power plants." Report No. NUREG/CR-4334; UCID-20444, Lawrence Livermore National Lab., CA (USA).

Collet, J. (1996). "Some remarks on rare-event approximation.” IEEE Transactions on Reliability, 45(1), 106-108.

Commission, C. N. S. et al. (2005). Probabilistic Safety Assessment (PSA) for Nuclear Power Plants. Canadian Nuclear Safety Commission.

de Angelis, M., Patelli, E., and Beer, M. (2015). “Advanced line sampling for efficient robust reliability analysis." Structural safety, 52, 170-182.

Faes, M., Sadeghi, J., Broggi, M., de Angelis, M., Patelli, E., Beer, M., and Moens, D. (2019). “On the robust estimation of small failure probabilities for strong non-linear models." ASCE-ASME Journal of Risk and Uncertainty in Engineering Systems Part B: Mechanical Engineering. 
Feng, G., Patelli, E., Beer, M., and Coolen, F. P. (2016). “Imprecise system reliability and component importance based on survival signature." Reliability Engineering \& System Safety, 150, 116-125.

Ferson, S., Kreinovich, V., Ginzburg, L., Myers, D. S., and Sentz, K. (2003). "Constructing probability boxes and dempster-shafer structures." Report No. SAND2002-4015, Sandia National Laboratories.

Jiang, C., Zheng, J., and Han, X. (2017). "Probability-interval hybrid uncertainty analysis for structures with both aleatory and epistemic uncertainties: a review." Structural and Multidisciplinary Optimization, 1-18.

Kaplan, S., Bier, V. M., and Bley, D. C. (1994). "A note on families of fragility curves - is the composite curve equivalent to the mean curve?." Reliability Engineering \& System Safety, 43(3), $257-261$.

Kapur, K. and Lamberson, L. (1977). Reliability in engineering design. Wiley.

Karanki, D. R., Kushwaha, H. S., Verma, A. K., and Ajit, S. (2009). "Uncertainty analysis based on probability bounds (p-box) approach in probabilistic safety assessment." Risk Analysis, 29(5), $662-675$.

Kennedy, R. P. (2011). "Performance-goal based (risk informed) approach for establishing the sse site specific response spectrum for future nuclear power plants." Nuclear Engineering and Design, 241(3), 648-656.

Kennedy, R. P., Cornell, C., Campbell, R., Kaplan, S., and Perla, H. (1980). "Probabilistic seismic safety study of an existing nuclear power plant." Nuclear Engineering and Design, 59(2), 315338.

Kim, J. H., Choi, I.-K., and Park, J.-H. (2010). "Seismic evaluation by using composite standard deviation." Transactions of the Korean Nuclear Society Autumn Meeting, Jeju, Korea (10).

Le Duy, T. D., Vasseur, D., Dieulle, L., Bérenguer, C., and Couplet, M. (2010). "Representation of parameter uncertainty with evidence theory in probabilistic risk assessment." Proceeding of the Workshop on the Theory of Belief Functions, Brest, France.

Modarres, M., Kaminskiy, M. P., and Krivtsov, V. (2016). Reliability engineering and risk analysis: 
a practical guide. CRC press.

Modarres, M. and Kim, I. S. (2010). Handbook of Nuclear Engineering. Springer US, Boston, MA, Chapter Deterministic and Probabilistic Safety Analysis, 1739-1812.

Moore, R. E., Kearfott, R. B., and Cloud, M. J. (2009). Introduction to interval analysis. Society for Industrial and Applied Mathematics.

Patelli, E. (2016). Handbook of Uncertainty Quantification. Springer International Publishing, Cham, Chapter COSSAN: A Multidisciplinary Software Suite for Uncertainty Quantification and Risk Management, 1-69.

Patelli, E., Alvarez, D. A., Broggi, M., and de Angelis, M. (2014). "An integrated and efficient numerical framework for uncertainty quantification: application to the NASA Langley multidisciplinary uncertainty quantification challenge." 16th AIAA Non-Deterministic Approaches Conference (SciTech 2014), AIAA SciTech, American Institute of Aeronautics and Astronautics.

Patelli, E., Alvarez, D. A., Broggi, M., and de Angelis, M. (2015). "Uncertainty management in multidisciplinary design of critical safety systems." Journal of Aerospace Information Systems, $12,140-169$.

Patelli, E., Broggi, M., Tolo, S., and Sadeghi, J. (2017a). "Cossan software: A multidisciplinary and collaborative software for uncertainty quantification." Proceedings of the 2nd ECCOMAS thematic conference on uncertainty quantification in computational sciences and engineering, UNCECOMP 2017, Rhodes Island, Greece.

Patelli, E., Feng, G., Coolen, F., and Coolen-Maturi, T. (2017b). "Simulation methods for system reliability using survival signature." Reliability Engineering \& System Safety, 167, 327-337.

Patelli, E., Pradlwarter, H. J., and Schuëller, G. I. (2011). “On multinormal integrals by importance sampling for parallel system reliability." Structural Safety, 33, 1-7.

Pellissetti, M., Keßler, H., Laudarin, F., Altieri, D., and Patelli, E. (2017). "Statistical analysis of impact forces and permanent deformations of fuel assembly spacer grids in the context of seismic fragility." Transactions, SMiRT-24.

Prinja, N. K., Ogunbadejo, A., Sadeghi, J., and Patelli, E. (2017). "Structural reliability of pre- 
stressed concrete containments.” Nuclear Engineering and Design, 323C, 235-244.

Qiu, Z., Yang, D., and Elishakoff, I. (2008). "Probabilistic interval reliability of structural systems.” International Journal of Solids and Structures, 45(10), 2850-2860.

Rüschendorf, L. (1991). "Fréchet-bounds and their applications." Advances in probability distributions with given marginals, 151-187.

Samaniego, F. J. (2007). System signatures and their applications in engineering reliability, Vol. 110. Springer Science \& Business Media.

Sun, H.-1. and Yao, W.-x. (2008). “The basic properties of some typical systems' reliability in interval form.” Structural Safety, 30(4), 364-373.

Sundararajan, C. R. (2012). Probabilistic structural mechanics handbook: theory and industrial applications. Springer Science \& Business Media. 


\section{List of Tables}

1 Input parameters for the modified Sandia National Laboratories containment test

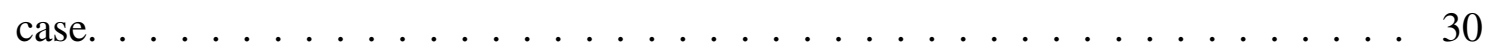

2 Input parameters for Sandia National Laboratories containment test case with additive component strengths. . . . . . . . . . . . . . . . . . . . 31 


\begin{tabular}{|l|l|l|}
\hline Failure Mode & $\begin{array}{l}\text { Logarithmic Median, } \mu, \\
\log \beta / \mathrm{MPa}\end{array}$ & $\begin{array}{l}\text { Logarithmic Standard } \\
\text { Deviation, } \sigma / \mathrm{MPa}\end{array}$ \\
\hline \hline Liner tear around personnel airlock & -0.0943 & {$[0,0.0017]$} \\
\hline Basemat shear & -0.0141 & {$[0,0.0016]$} \\
\hline Cylinder hoop membrane & 0.0853 & {$\left[0,8.8641 \times 10^{-4}\right]$} \\
\hline Wall-basemat junction shear & 0.1231 & {$[0,0.0014]$} \\
\hline Cylinder meridional membrane & 0.2159 & {$\left[0,8.3320 \times 10^{-4}\right]$} \\
\hline Dome membrane & 0.5911 & {$\left[0,5.345 \times 10^{-4}\right]$} \\
\hline Personnel air lock door buckling & 0.2159 & {$[0,0.0013]$} \\
\hline
\end{tabular}

TABLE 1. Input parameters for the modified Sandia National Laboratories containment test case. 


\begin{tabular}{|c|c|c|}
\hline Load and Strength & $\begin{array}{l}\text { Mean } \quad \text { Value, } \\
{[\underline{\mu,}, \bar{\mu}]}\end{array}$ & Coefficient of Variation \\
\hline Concrete tensile strength, $F_{c}$ & {$[4.3,4.5]$} & 0.2 \\
\hline Liner yield, $F_{l}$ & {$[370,390]$} & 0.2 \\
\hline Rebar yield, $F_{s}$ & {$[450,370]$} & 0.2 \\
\hline Tendon yield, $F_{t}$ & {$[1700,1800]$} & 0.2 \\
\hline Design Pressure, $P_{d}$ & 0.39 & 0.2 \\
\hline Radius, $R$ & 5537.5 & 0.2 \\
\hline Concrete area, $A_{c}$ & 312.85 & 0.2 \\
\hline Liner area, $A_{l}$ & 1.6 & 0.2 \\
\hline Rebar area, $A_{s}$ & 6.85 & 0.2 \\
\hline Tendon area, $A_{t}$ & 3.7 & 0.2 \\
\hline
\end{tabular}

TABLE 2. Input parameters for Sandia National Laboratories containment test case with additive component strengths. 


\section{List of Figures}

1 The composite curve compared to the median curve $\left(\beta=\beta_{e}\right.$ and $\left.\sigma=\sigma_{a}\right)$, and the curves with 5th and 95th percentiles of $\beta$ and $\sigma=\sigma_{a}$. In the example $\sigma_{a}=0.2$,

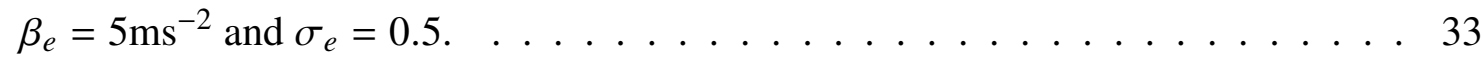

2 A comparison of extreme fragility curves enclosed within the fragility probability box. The parameters for the plotted probability box were $\underline{\mu}=\log \underline{\beta}=1 \mathrm{~ms}^{-2}$, $\bar{\mu}=\log \bar{\beta}=1.2 \mathrm{~ms}^{-2}, \underline{\sigma}=0.2$ and $\bar{\sigma}=0.5 \ldots \ldots \ldots \ldots$

3 Demonstration of failure probability calculation with Eqn. (16). The lognormal probability density functions for the stress and strength are shown. The shaded area represents the integrand in Eqn. (15), which yields the failure probability $P_{f}=0.14$. The example parameter values for the plotted distributions were $\beta_{l}=1 \mathrm{~ms}^{-2}, \sigma_{l}=1, \beta_{i}=3 \mathrm{~ms}^{-2}$ and $\sigma_{i}=0.2 \ldots \ldots \ldots \ldots$

4 Demonstration of failure probability calculation with Eqn. (18). The lognormal probability density functions for the stress and strength are shown. The shaded area represents the integrand in Eqn. (15), which yields the failure probability $P_{f}=0.12$. The example parameter values for the plotted distributions were $K_{H}=2, k_{1}=1\left(\mathrm{~ms}^{-2}\right)^{K_{H}}, \beta_{i}=3 \mathrm{~ms}^{-2}$ and $\sigma_{i}=0.2 \ldots \ldots \ldots$

5 Probability box representing the fragility curve of the series system, computed analytically. For comparison, the results of a double loop Monte Carlo simulation are shown, which was computed by making 100 epistemic samples and 1000 aleatory samples. . . . . . . . . . . . . . . . . 37 


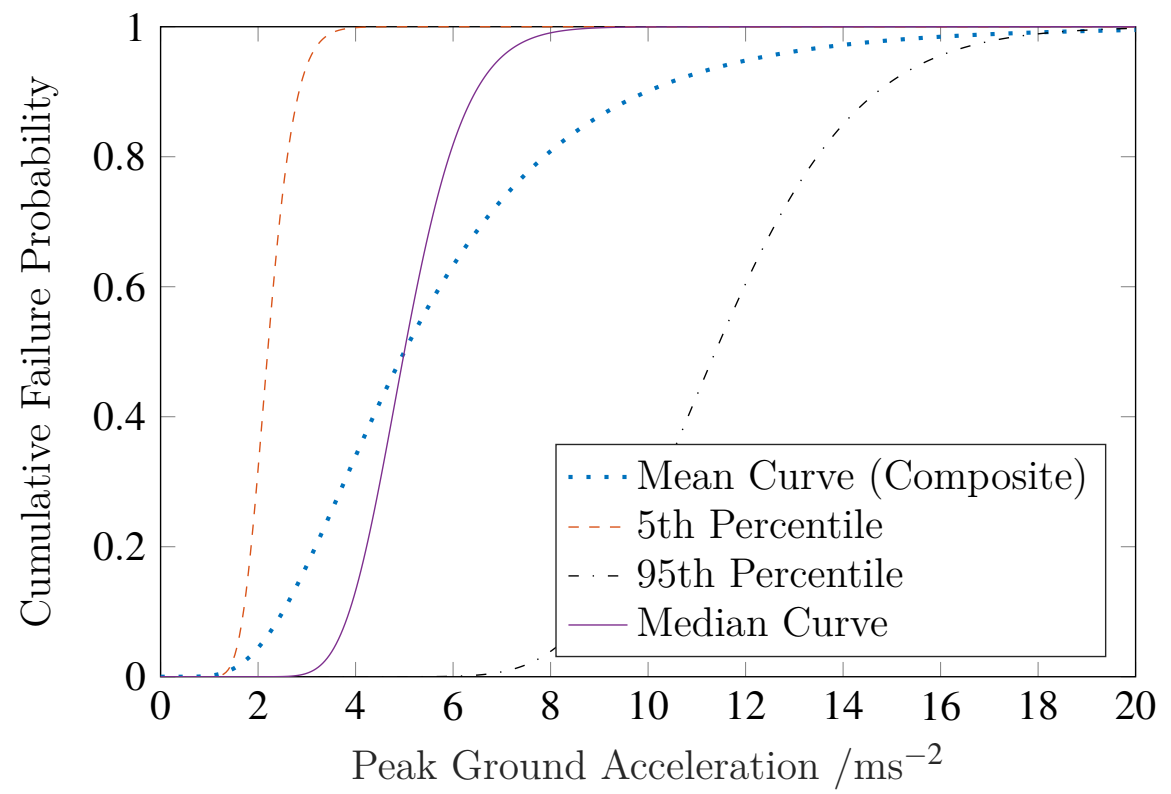

Fig. 1. The composite curve compared to the median curve $\left(\beta=\beta_{e}\right.$ and $\left.\sigma=\sigma_{a}\right)$, and the curves with 5th and 95th percentiles of $\beta$ and $\sigma=\sigma_{a}$. In the example $\sigma_{a}=0.2, \beta_{e}=5 \mathrm{~ms}^{-2}$ and $\sigma_{e}=0.5$. 


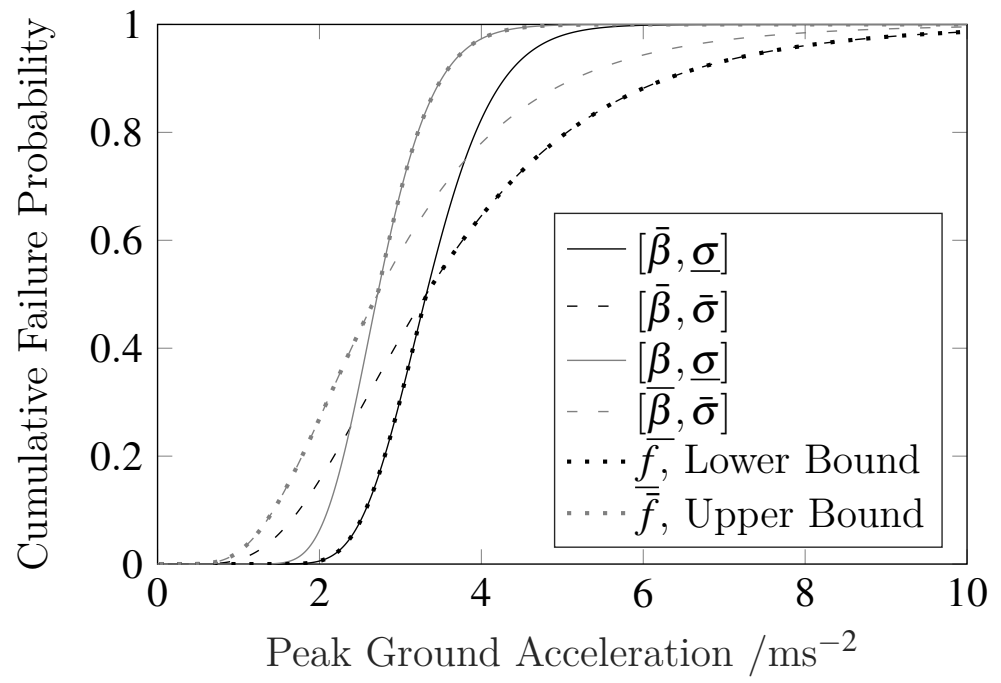

Fig. 2. A comparison of extreme fragility curves enclosed within the fragility probability box. The parameters for the plotted probability box were $\mu=\log \beta=1 \mathrm{~ms}^{-2}, \bar{\mu}=\log \bar{\beta}=1.2 \mathrm{~ms}^{-2}, \underline{\sigma}=0.2$ and $\bar{\sigma}=0.5$. 


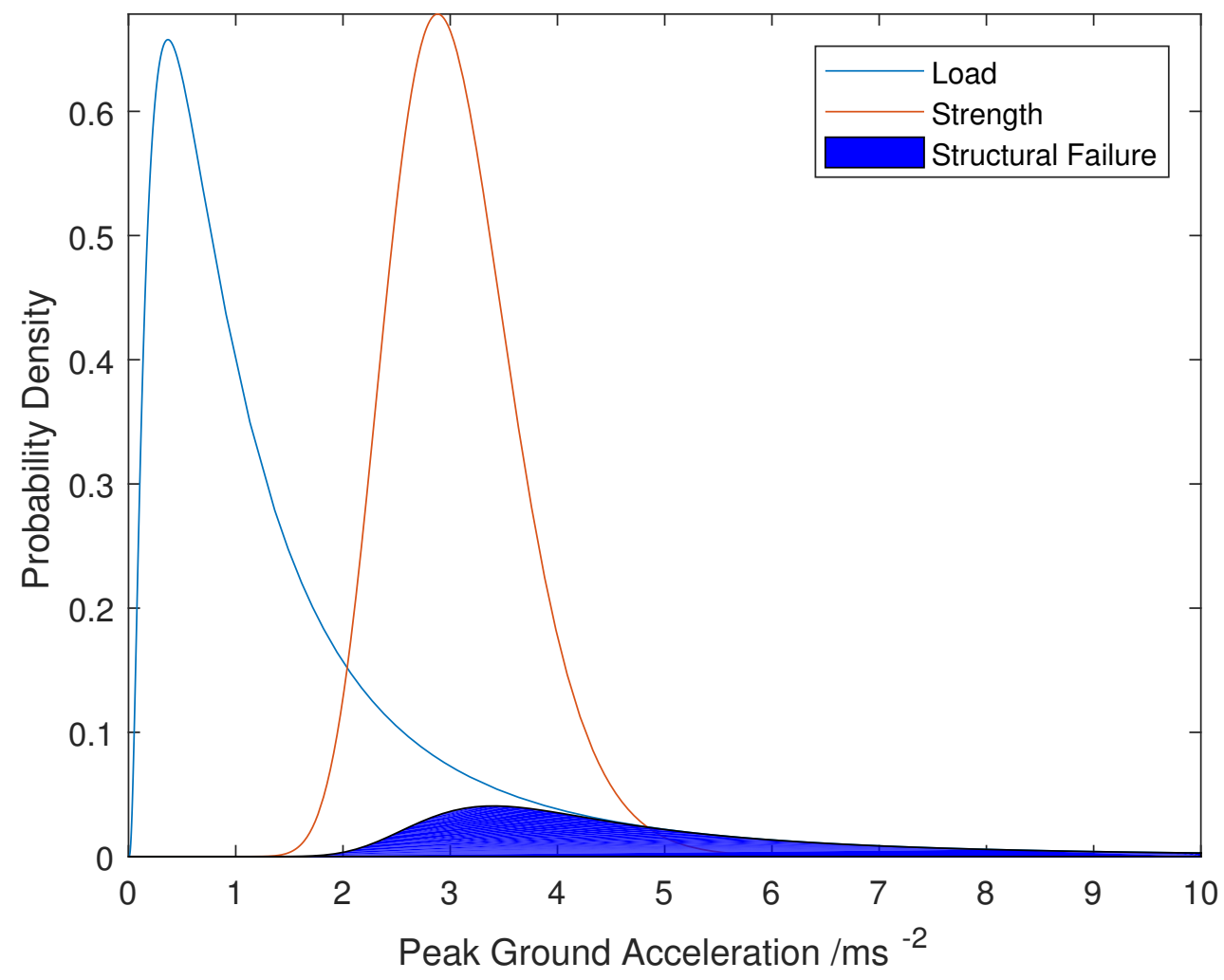

Fig. 3. Demonstration of failure probability calculation with Eqn. (16). The lognormal probability density functions for the stress and strength are shown. The shaded area represents the integrand in Eqn. (15), which yields the failure probability $P_{f}=0.14$. The example parameter values for the plotted distributions were $\beta_{l}=1 \mathrm{~ms}^{-2}, \sigma_{l}=1, \beta_{i}=3 \mathrm{~ms}^{-2}$ and $\sigma_{i}=0.2$. 


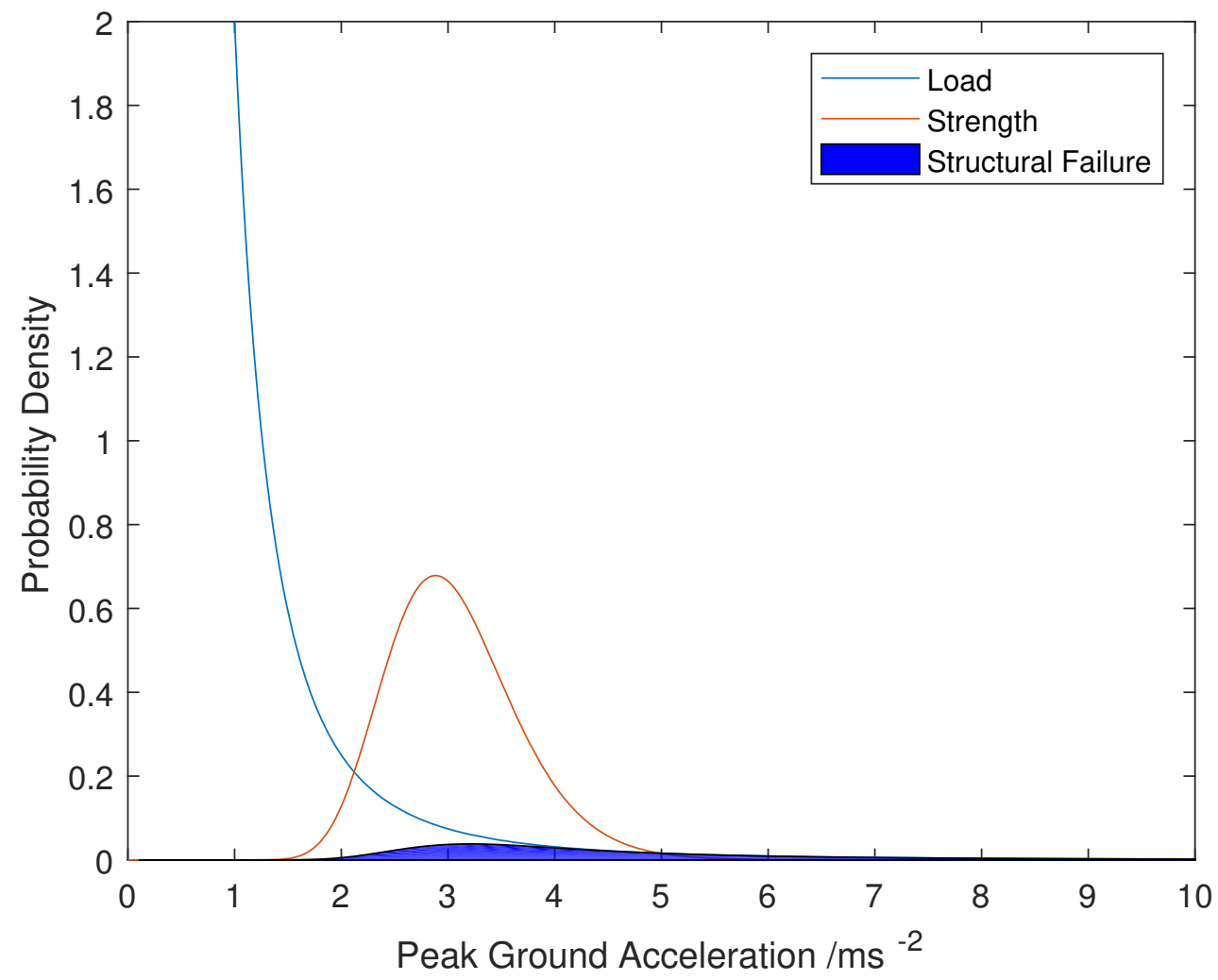

Fig. 4. Demonstration of failure probability calculation with Eqn. (18). The lognormal probability density functions for the stress and strength are shown. The shaded area represents the integrand in Eqn. (15), which yields the failure probability $P_{f}=0.12$. The example parameter values for the plotted distributions were $K_{H}=2, k_{1}=1\left(\mathrm{~ms}^{-2}\right)^{K_{H}}, \beta_{i}=3 \mathrm{~ms}^{-2}$ and $\sigma_{i}=0.2$. 


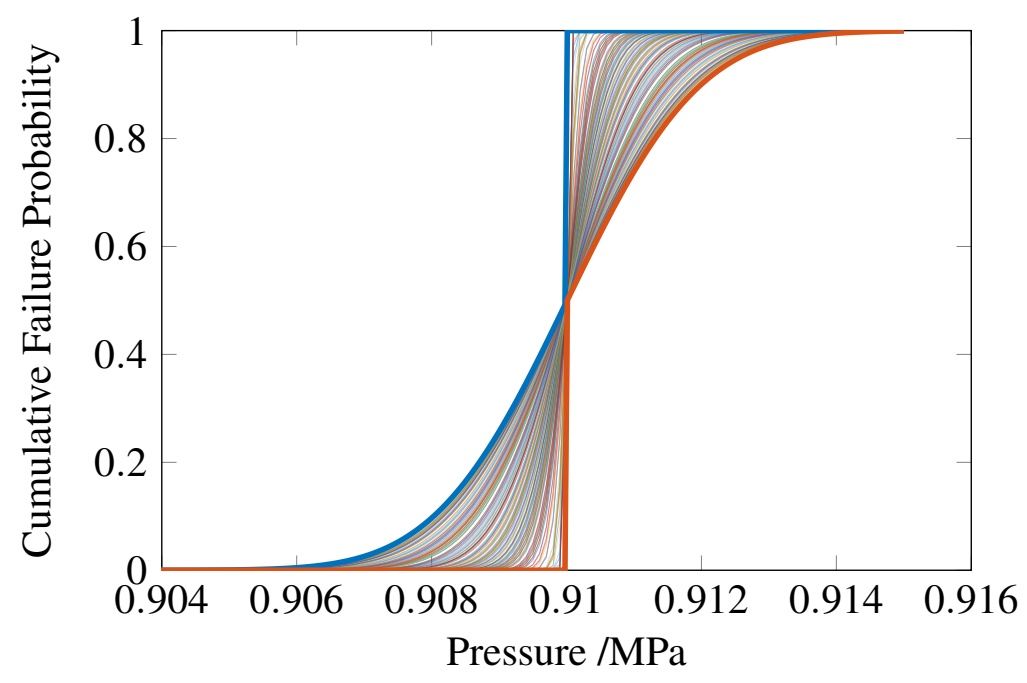

Fig. 5. Probability box representing the fragility curve of the series system, computed analytically. For comparison, the results of a double loop Monte Carlo simulation are shown, which was computed by making 100 epistemic samples and 1000 aleatory samples. 